Research Article

\title{
Decision-Making Approach of Two-Sided Matching of Supply and Demand of Logistics Service Based on the Uncertain Preference Ordinal
}

\author{
Na Wang $\mathbb{B D}^{1,2}{ }^{1,}$ Yinzhen Li, $^{1}$ and Cunjie Dai ${ }^{1}$ \\ ${ }^{1}$ School of Traffic and Transportation, Lanzhou Jiaotong University, Lanzhou, China \\ ${ }^{2}$ School of Mechatronic Engineering, Lanzhou Jiaotong University, Lanzhou, China \\ Correspondence should be addressed to Na Wang; 593215334@qq.com
}

Received 15 March 2020; Revised 20 July 2020; Accepted 19 August 2020; Published 14 October 2020

Academic Editor: Francesco Zammori

Copyright (c) $2020 \mathrm{Na}$ Wang et al. This is an open access article distributed under the Creative Commons Attribution License, which permits unrestricted use, distribution, and reproduction in any medium, provided the original work is properly cited.

\begin{abstract}
Aiming at the problem of matching logistics service supply and demand, this paper proposes a two-sided matching decision model of logistics service supply and demand based on the uncertain preference ordinal. In this model, the uncertain preference ordinal information is first expressed by the interval numbers of the logistics service supply and demand, and it is converted into the satisfaction degree of supply and demand matching uncertainty expressed by the interval number. Then, a multiobjective optimal matching model is constructed based on the largest overall satisfaction of the logistics service supply and demand side and the smallest satisfaction variance of the supply- and demand-side individual, and the multiobjective solution algorithm is designed based on nondominated sorting genetic algorithm-III (NSGA-III). Interval numbers are used to sort the matching results to obtain the approximately optimal two-sided matching scheme. Finally, this paper verifies the correctness of the model and validity of the algorithm.
\end{abstract}

\section{Introduction}

As we all know, theory comes from life practice. We are making choices all the time, from the marriage matching choice of men and women to the choice of positions by university graduates, and we constantly choose in order to obtain satisfactory results for ourselves or the group, that is to say, where there is a choice, there is matching.

In 1962, American economists Lloyd Shapley and David Gale published the foundational paper College Admissions and the Stability of Marriage; this paper analyzed the marriage problems, and it is proved that, under the strict preference algorithm (Gale-Shapley algorithm), each applicant will produce the optimal stable matching. The conclusion is that there is nonempty stable matching in the marriage model [1]. This research is regarded as the pioneering work of the two-sided matching theory. It has been developed for more than 50 years since its establishment. Subsequently, it has attracted the attention of scholars in many fields and continuously improved the theory and algorithms.

The rapid development of the logistics service industry helps to improve product transportation efficiency, reduce enterprise logistics costs, and enhance the company's core competitiveness. In the fierce competition, the goal of the logistics service demand side is to obtain the most satisfactory logistics service at the lowest cost, and the goal of the logistics service supply side is to obtain the satisfaction of the logistics demand side while obtaining relatively high profits. The transaction relationship between supply and demand parties is a typical noncrossing relationship, and the matching decision of the two conforms to the voluntary and voluntary market operation. Therefore, the cooperation characteristics of the supply and demand sides of the logistics service meet the basic requirements of the two-sided matching theory, which is suitable for the research and analysis of the two-sided matching theory and matching decision-making methods. 
In the research on the decision-making of two-sided matching of supply and demand of logistics service, the supply side and the demand side will make a comprehensive evaluation on the matchable object according to respective different demand and matching indicators. However, during the actual decision-making process, the decision maker cannot provide the preference ordinal information of each matching object and generally set the preference ordinal within a range so that the preference ordinal information generated has uncertainty. Therefore, the two-sided matching decision-making problem based on uncertain preference ordinal information has more wide applications in the actual decision-making problem.

Due to the complexity of the demand of the supply side and the demand side and the matching indicators, it is hard to make a decision with certain preference ordinal information to the matching object of the supply side and the demand side. The research on the two-sided matching problem based on uncertain preference ordinal has raised considerable concerns. Qi and Fan [2] introduced the concept of matching satisfaction and payment, established the multiobjective optimization model of the two-sided matching decision-making problem based on uncertain preference ordinal information, and adopted the weighted solution model based on the membership function. Qi et al. [3] provided the two-sided matching problem description considering the psychological behavior of the subject under uncertain preference ordinal information and constructed the two-objective optimization model. Kayvanfar et al. [4] proposed an intelligent water drops (IWD) algorithm for multilevel supply chain optimization problems, which minimized the cost of logistics and improved the overall level of customer service. Sun et al. [5] transformed the uncertain preference ordinal and other two forms of preference information into an ordinal value vector according to risk functions and other theories and built an optimization model of two-sided matching satisfaction. Kayvanfar et al. [6] aimed at minimizing logistics costs and studied the twostage stochastic programming problem with integers and binary first-stage and continuous second-stage decision variables. The model comprehensively considers the uncertain customer demand, the manufacturer's production capacity, and the supplier's supply capacity. Fan et al. [7] calculated the expected preference ordinal based on the uncertain preference ordinal of each agent to the relative agent by considering the psychological factors and obtained the possible matching result according to the disappointment theory. Kayvanfar et al. [8] aimed at a specific public warehouse (called SDHIC) managed by third-party logistics to deal with the problem of the supply chain of integrated circuits and proposed a practical mathematical processing method that optimizes the total logistics cost. Qi [9] considered the two-sided matching problem with uncompleted score acquiring information and put forward a kind of twosided matching method. Zhang et al. [10], according to the thought of the traditional Borda approach concerning the decision-making problem of two-sided matching based on the uncertain preference ordinal, put forward a decisionmaking approach. You et al. [11] put forward a kind of assignment approach of solving group decision-making problems under uncertain preference information. Liu and Ma [12] put forward a kind of approach of calculating that each matching solves the two-sided matching problem based on the uncertain preference ordinal to the preference distance and solved in the branch-bound algorithm. Kayvanfar et al. [13] established a quantitative model for supply chain management and integrated circuits. The model aims to minimize logistics costs (transportation and order costs) and future logistics costs (inventory and out-of-stock costs). Kayvanfar et al. [8] proposed a new mathematical method to be applied to the supply chain (SC) ICs' joint provision of warehousing activities called SDHIC. This model proposes a heuristic algorithm based on linear relaxation, which can quickly produce initial feasible solutions. Tirkolaee et al. [14] proposed a hybrid selection method based on fuzzy logic for the problem of sustainable and reliable supplier selection in a two-level supply chain. Kayvanfar et al. [15] applied the concept of supply-demand hub in ICs (SDHIC) to optimize the way of enterprise interaction in industrial clusters. In order to evaluate the values of SDHIC, three mathematical models are proposed, and a heuristic algorithm based on the linear theory is designed, which can solve the model quickly and effectively. Verification by examples shows that SDHIC has better applicability to small- and medium-sized enterprises in actual use.

There are research studies that have provided reference and guidance in theoretical and methodological aspects for the two-sided matching of supply and demand of logistics service. However, in the above studies, there are few studies on two-sided matching of uncertain preference ordinal. In the research, the matching subjects are mainly considered from the perspective of overall satisfaction, while the satisfaction and balance of individuals in the matching subjects are not involved. When individual satisfaction is not fully satisfied, it is difficult to guarantee the fairness and stability of matching. In the case of the maximum overall satisfaction, the satisfaction of the individuals in the two-sided matching subject is considered in the matching process. Only when matching satisfaction of the individuals in the subject is more balanced can a stable and fair two-sided matching be achieved. Given this, this paper considers to express the uncertain preference information of the matching subjects of supply and demand of logistics service by the operation of interval numbers and turn it into uncertain satisfaction of supply and demand with the maximum overall uncertainty satisfaction of the supply side and the demand side of logistics service and the minimum individual uncertainty satisfaction variance of the supply side and the demand side, builds the multiobjective optimization model of two-sided matching of supply and demand of multi-to-multi logistics service based on uncertain preference ordinal, and designs the solving algorithm of the multiobjective model based on NSGA-III.

This paper takes logistics service supply and demand information as data sources and systematically studies the matching problem between logistics service suppliers and logistics service demanders in logistics activities. The research mainly contributes to the state of the art in the 
following: firstly, this paper studies the supply-demand matching problem of multi-to-multi logistics services, which provides new research ideas and references for the study of multi-to-multi two-sided matching theory. Secondly, the optimization model considers both the overall satisfaction of the logistics service matching parties and the balance of the individual satisfaction of the logistics service matching parties, highlighting the matching fairness. Thirdly, considering the uncertainty of individual preference ordinal information of supply and demand sides in reality, this paper uses interval number theory and interval number comparison method to clear the uncertainty problem and obtains effective calculation results to solve the two-sided matching problem of logistics service supply and demand, which makes the logistics service supply and demand problem more suitable for the actual needs. Fourthly, the logistics service supply side and the logistics service demand side can obtain a satisfactory and fair match, which shortens the operation cycle of the logistics service activity, which has a great impetus for the development of the logistics market.

The rest of this paper is organized as follows: Section 2 covers the problem description; Section 3 covers the decisionmaking model of two-sided matching of supply and demand of logistics service; Section 4 studies the multiobjective solution algorithm based on NSGA-III; Section 5 covers the calculation example analysis; and finally, conclusions and future research directions are given in Section 6.

\section{Problem Description}

In the decision-making approach of two-sided matching of supply and demand of logistics service based on uncertain preference ordinal, assume that matching subject $A$ is the demand side of logistics service, and its set is $A=\left\{A_{1}, A_{2}, A_{3}, \ldots, A_{m}\right\}$, wherein $A_{i} \in A$ represents the $i$-th subject of the demand side, and $A_{i} \in A=\{1,2,3, \ldots, m\}$; assume that matching subject $B$ is the supply side, and its set is $B=\left\{B_{1}, B_{2}, B_{3}, \ldots, B_{n}\right\}$, wherein $B_{j}$ represents the $j$-th subject of the supply side, $B_{j} \in J=\{1,2, \ldots, n\}$.

Assume that the preference vector of the uncertain ordinal preference of the demand side $A_{i}$ related to the supply side set $B$ is $\widetilde{O c_{i}}=\left\{\widetilde{o c_{i 1}} \widetilde{o c_{i 2}}, \ldots, \widetilde{o c_{i n}}\right\}$, wherein $\widetilde{o c_{i j}}=$ $\left[o c_{i j}^{l}, o c_{i j}^{u}\right]$ represents that the demand side $A_{i}$ ranks the supply side $B_{j}$ between $o c_{i j}^{l}$-th and $o c_{i j}^{u}$-th, wherein $o c_{i j}^{l} \in I, o c_{i j}^{u} \in I$, and $o c_{i j}^{l} \leq o c_{i j}^{u}$; assume that the preference vector of the uncertain value of the supply side $B_{j}$ related to the demand side set $A$ is $\widetilde{O d_{j}}=\left\{\widetilde{o d_{1 j}} \widetilde{o d_{2 j}}, \ldots, \widetilde{o d_{m j}}\right\}$, wherein $\widetilde{o d_{i j}}=\left[o d_{i j}^{l}, o d_{i j}^{u}\right]$ represents that the supply side $B_{j}$ ranks the demand side $A_{i}$ between $o d_{i j}^{l}$-th and $o d_{i j}^{u}$-th, wherein $o d_{i j}^{l} \in J$, $o d_{i j}^{u} \in J$, and $o d_{i j}^{l} \leq o d_{i j}^{u}$.

This article intends to address the preference ordinal of $\widetilde{O c_{i}}$ and $\widetilde{O d_{j}}, i \in I, j \in J$; the optimal matching scheme is selected by using the two-sided matching decision method of logistics service supply and demand based on an uncertain preference ordinal. In the research, the uncertain preference ordinal $\widetilde{O c_{i}}$ given by individual $A_{i}$ on the demand side and the uncertain preference ordinal $\widetilde{O d_{j}}$ given by individual $B_{j}$ on the supply side turn them into demand-side $A_{i}$ uncertain satisfaction $\widetilde{C}_{i}=\left\{\widetilde{c}_{i 1}, \widetilde{c}_{i 2}, \ldots, \widetilde{c}_{i n}\right\}$, wherein $\widetilde{c}_{i j}=\left[c_{i j}^{l}, c_{i j}^{u}\right]$, and supply-side $B_{j}$ uncertain satisfaction $\widetilde{D}_{j}=\left\{\widetilde{d}_{1 j}, \widetilde{d}_{2 j}, \ldots, \widetilde{d}_{m j}\right\}$, wherein $\tilde{d}_{i j}=\left[d_{i j}^{l}, d_{i j}^{u}\right]$. Therefore, a multiobjective optimal matching model of the two-sided matching decision of logistics service supply and demand based on the uncertain preference ordinal is established; then, matching supply and demand through a two-sided decision method with uncertain preference ordinal, a relatively optimal solution is obtained.

From the expression mode of the uncertain ordinal value preference vector given between the supply side and the demand side, we can know that the value of the preference vector is within a given closed interval and is not subject to some probability distribution, so it cannot be expressed as a random number; there is neither a membership degree related to it, so it cannot be expressed with a fuzzy number. Combined with the form of interval number and its relevant theory, the interval number can be used to carry out the quantization and calculation of the uncertain preference vector. From the rankings when the supply side and the demand side make a selection, we can know that the smaller the interval number, the higher the ranking and the higher the selected preference degree.

2.1. Relative Theory of the Interval Number. The interval number was put forward by Moore in the 1960s. The interval arithmetic constructed based on the interval number cannot only process the imprecise data of the vector participating in calculation but also keep track of the truncation error and round-off error automatically; meanwhile, it has the advantages of simple calculation and less data quantity required, and it is not necessary to consider the distribution characteristics of the uncertain vector in presentation and overcome the deficiencies that the probabilistic approach processes uncertainty. The interval analysis approach of the uncertain problems has become the hot spot of the engineering technology field, management decision-making field, and theoretical research at present.

Interval number is the number presented by the internal, it is a set composed of all real numbers on a closed interval, and there are detailed definition and calculation rules in the document $[16,17]$, for example, the relevant calculation formula of exponential and logarithmic operations and power and square operations has been provided. After carrying out the operation on the interval number, the calculation result is still the interval number. If decisionmaking is required according to the calculation result of the interval number, it is necessary to compare the size of the interval number. Be different from the way of comparison of the size between the certain real number, when comparing the size of the interval number $\widetilde{a}$ and $\widetilde{b}$, or ranking the interval number $\widetilde{a}$ and $\widetilde{b}$, some special ways are required to process the data.

Based on the two-dimensional relationship of interval numbers, Zhang and Zhang [18] provided the possibility degree formula of comparison of the size of the interval number: 


$$
P(\widetilde{a}<\tilde{b})= \begin{cases}0, & b^{l} \leq b^{u} \leq a^{l}<a^{u}, \\ \frac{\left(b^{u}-a^{l}\right)^{2}}{2\left(a^{u}-a^{l}\right)\left(b^{u}-b^{l}\right)}, & b^{l}<a^{l}<b^{u}<a^{u}, \\ 1-\frac{a^{l}+a^{u}-2 b^{l}}{2\left(b^{u}-b^{l}\right)}, & b^{l} \leq a^{l} \leq a^{u}<b^{u}, \\ 1-\frac{2 a^{u}+b^{u}-b^{l}}{2\left(a^{u}-a^{l}\right)}, & a^{l} \leq b^{l} \leq b^{u} \leq a^{u}, \\ 1-\frac{\left(a^{u}-b^{l}\right)^{2}}{2\left(a^{u}-a^{l}\right)\left(b^{u}-b^{l}\right)}, & a^{l}<b^{l} \leq a^{u} \leq b^{u}, \\ 1, & \end{cases}
$$

This expression can present the possibility degree when two interval numbers make comparison according to different values of infimum and supremum of the two interval numbers; in the further calculation of this paper, equation (1) is used to make comparison and rankings of the size of the interval number.

\section{Decision-Making Model of Two-Sided Matching of Supply and Demand of Logistics Service}

The traditional decision-making approach of two-sided matching is to carry out decision-making according to the preference value of the influence of each influence factor in the subject to another subject. In the existing research, it is always the preference information given by subject $A$ to subject $B$, but in the face of the actual problem, the acquisition of these preference information is always difficult or uncertain, and the preference will change along with the environment, requirements, and individual difference. Both parties of the body have their evaluation criteria, which require the authoritative intermediaries to match with the other side's actual satisfaction after knowing the respective expectation of the subject and the actual standard when both sides of the body provide their expected information, and the two-sided matching result can be obtained according to the decision-making approach of uncertain preference ordinal.

3.1. Construction of the Decision-Making Model. In the twosided decision-making approach of supply and demand of logistics service based on uncertain preference ordinal information, it is necessary to transform the known uncertain preference ordinal information into the corresponding uncertain satisfaction of the supply side and the demand side. Assume that, after the transformation, the uncertain satisfaction of supply subject $A_{i}$ to demand subject $B_{j}$ is $\tilde{c}_{i j}$, and the uncertain satisfaction of demand subject $B_{j}$ to supply subject $A_{i}$ is $\tilde{d}_{i j}$. Assume that $x_{i j}$ is the variable of $0-1$, wherein $x_{i j}=0$ represents $A_{i}$ do not match $B_{j}$ and $x_{i j}=1$ represents $A_{i}$ do match $B_{j}$. Under the premise of calculating the maximum overall satisfaction of two-sided matching of supply and demand of multi-to-multi logistics service, we consider the size of the variance of individual satisfaction of the demand side $A_{i}$ and the supply side $B_{j}$ of logistics service in two-sided matching, making $\widetilde{f}_{A}=\left(\sum_{i} \sum_{j} \widetilde{c}_{i j} x_{i j} / m\right)$ to be the average value of the satisfaction of the demand side of logistics service based on the uncertain preference ordinal and making $\widetilde{f}_{B}=\left(\sum_{i} \sum_{j} \tilde{d}_{i j} x_{i j} / n\right)$ to be the average value of the satisfaction of the supply side of logistics service based on the uncertain preference ordinal.

According to the satisfaction vector $M=\left[\widetilde{c}_{i j}\right]_{m \times n}$ and $W=\left[\tilde{d}_{i j}\right]_{m \times n}$, the multiobjective optimization model of twosided matching of supply and demand of logistics service based on the uncertain preference ordinal can be established to be

$$
\begin{aligned}
& \max Z_{1}=\sum_{i=1}^{m} \sum_{j=1}^{n} \tilde{c}_{i j} x_{i j}, \\
& \min Z_{2}=\frac{\sum_{i=1}^{m}\left(\sum_{j=1}^{n} \tilde{c}_{i j} x_{i j}-\widetilde{f}_{A}\right)}{\sum_{i} \sum_{j} x_{i j}} \\
& \max Z_{3}=\sum_{i=1}^{m} \sum_{j=1}^{n} \tilde{d}_{i j} x_{i j}, \\
& \min Z_{4}=\frac{\sum_{i=1}^{m}\left(\sum_{j=1}^{n} \tilde{d}_{i j} x_{i j}-\tilde{f}_{B}\right)}{\sum_{i} \sum_{j} x_{i j}} \\
& \text { s.t. } \sum_{j=1}^{n} x_{i j} \leq m, \quad i=1,2, \ldots, m, \\
& \sum_{i=1}^{m} x_{i j} \leq n, \quad j=1,2, \ldots, n, \\
& \sum_{j=1}^{n} x_{i j} \geq 1, \quad i=1,2, \ldots, m, \\
& \sum_{i=1}^{m} x_{i j} \geq 1, \quad j=1,2, \ldots, n \\
& x_{i j}=\{0,1\}, \quad i=1,2, \ldots, m, j=1,2, \ldots, n .
\end{aligned}
$$

In the model, equations (2)-(5) are objective functions, and the implication of equation (2) is to maximize the satisfaction of the demand side $A_{i}$ of logistics service to the supply side of logistics service; equation (3) represents that the individual demand side $A_{i}$ of logistics service matches with the individual supply side $B_{j}$ of logistics service, and the 
variance of the individual satisfaction of $A_{i}$ is the minimum; the implication of equation (4) is to maximize the satisfaction of the supply side $B_{j}$ to the demand side of logistics service; equation (5) represents that the individual supply side $B_{j}$ of logistics service matches with the individual demand side $A_{i}$, and the variance of the individual satisfaction of $B_{j}$ is the minimum; equation (6) represents that each demand subject of logistics service can match with multiple supply subjects of logistics service; equation (7) represents that each supply subject of logistics service can match with multiple demand subjects of logistics service; the implication of equation (8) is that each supply side of logistics service matches with at least one demand subject of logistics service; the implication of equation (9) is that each demand side of logistics service matches with at least one supply subject of logistics service; and equation (10) represents the $0-1$ decision variable.

3.2. Analysis of Features of the Model. There are many optimization objectives in the model, and it is hard to unify the optimization direction between each objective. When comparing the advantages and disadvantages of the solution, it is usually determined by the dominant relation between the solutions.

Generally speaking, the problem of multiobjective optimization can be defined to optimize the objective function vector within the feasible region by determining a group of decision variables. Under the premise that assuming each objective vector taking minimum for optimization, the problem of multiobjective optimization takes the following form:

$$
\begin{array}{ll}
\min & z(\mathbf{x})=\left(f_{1}(\mathbf{x}), f_{2}(\mathbf{x}), \ldots, f_{H}(\mathbf{x})\right) \\
\text { s.t. } & g(\mathbf{x}) \leq 0, \mathbf{x} \in \mathbf{X} .
\end{array}
$$

In equation (11), $\mathbf{x} \in R^{n}$ is the vector of the decision variable; $\mathbf{X}=\{\mathbf{x}\}$ is a decision set of the feasible solution; $z(\mathbf{x})$ is a vector-valued function to be optimized; $H$ is the total quantity of the optimization objective; $f_{h}(\mathbf{x})(h=1,2, \ldots, H)$ is the objective function to be optimized; and $g(\mathbf{x})$ is a vector of constraint function.

The optimal solution of multiobjective optimization is usually defined by the dominance relation between the solutions. In the context of equation (11), the dominance relations of solutions have the following definitions.

Definition 1 (see [19]). Given $\mathbf{x}$ and $\mathbf{y}$ are the two feasible solutions in equation (11). If $f_{h}(\mathbf{x})<f_{h}(\mathbf{y})$ for all $h, \mathbf{x}$ is said to strictly dominate $\mathbf{y}$, say $\mathbf{x}<\mathbf{y}$ or $z(\mathbf{x})<z(\mathbf{y})$; if $f_{h}(\mathbf{x}) \leq f_{h}(\mathbf{y})$ for some $h$ in $H$ optimization objectives and if $f_{s}(\mathbf{x}) \leq f_{s}(\mathbf{y})$ for other optimization objectives $s, \mathbf{x}$ is said to dominate $\mathbf{y}$, say $\mathbf{x}<\mathbf{y}$ or $z(\mathbf{x})<z(\mathbf{y})$; if $f_{h}(\mathbf{x}) \leq f_{h}(\mathbf{y})$ for all $h$, $\mathbf{x}$ is said to weakly dominate $\mathbf{y}$, say $\mathbf{x}<\mathbf{y}$ or $z(\mathbf{x})<z(\mathbf{y})$; and if neither $\mathbf{x} \prec \mathbf{y}$ nor $\mathbf{y} \prec \mathbf{x}, \mathbf{x}$ and $\mathbf{y}$ are said to dominate each other, say $\mathbf{x} \cong \mathbf{y}$.

Definition 2 (see [20]). In set $\mathbf{X}$, if there is a solution $\mathbf{x} \in \mathbf{X}$ and for any other solution $\mathbf{y}$ in $\mathbf{X}$, there does not exist $\mathbf{y}<\mathbf{x}$, and $\mathbf{x}$ is called as a nondominated solution or Pareto-optimal solution; if the solutions in set $E$ are all efficient, set $E$ is called as the Pareto-optimal solution set or nondominant solution set.

Definition 3 (see [20]). If the solution $\mathbf{x}^{*}$ is a nondominated solution or the Pareto-optimal solution, $\mathbf{z}^{*}=\mathbf{z}\left(\mathbf{x}^{*}\right)$ is called as the nondominated point; set $F$ of a nondominated point is called as the nondominated frontier or the Pareto frontier.

The goal of the solution of the two-sided matching problem under uncertain conditions is to obtain the matching result corresponding to each solution in a nondominated solution set $E$ through calculating nondominated frontier $F$ corresponding to each solution in $E$, which enables the decision maker to determine the vector $\mathbf{x}$ in $E$ according to the value in $F$ to make a selection for the matching result under different objectives.

\section{Multiobjective Solution Algorithm Design}

In regard to the solution of the problem of multiobjective optimization, there are many proven and effective methods which are mainly divided into the accurate algorithm, heuristic algorithm, and meta-heuristic algorithm. Liu [21], in regard to the decision-making problem of two-sided matching with individual preference ordinal and combined preference ordinal information, established a multiobjective optimization model with the maximum matching satisfaction as the goal and designed multiobjective evolutionary algorithms to solve the model. Kong and Jiang [22] considered the problem of personnel who coordinate information and two-sided matching of the post, according to multi-indicator evaluation information provided by the personnel and the post, established the multiobjective optimization model of taking the maximum satisfaction of personnel to the post, the maximum satisfaction of the individual skill of the post to the personnel, and the maximum coordination between the personnel, and designed a multiobjective discrete differential evolutionary algorithm to solve the model. Yuan and Jiang [23], in regard to the oneto-multiple two-sided matching problem of the patients and surgeons of selective operation, according to the preference information provided by the surgeon to the type of operation and the preference information and aspiration level provided by the patients to the surgeon, constructed the multiobjective optimization model of obtaining the matching scheme of doctor-patient stability of satisfying the aspiration level and designed a heuristic algorithm of solving the model based on the nondominated sorting genetic algorithm, NSGA-II, with elitist strategy. Sangaiah et al. [24] proposed an EPPS mathematical model based on social media and big data-driven computing for the E-projects portfolio selection (EPPS) problem. A hybrid algorithm NSGA-III-MOIWO is proposed for this model. At the same time, the advantages of NSGA-III and MOIWO algorithm are used to deal with the complexity of the problem. Tirkolaee et al. [25] proposed a new type of biobjective mixedinteger linear programming model for the problem of FSS. In order to solve the model more effectively, a hybrid 
algorithm based on interactive fuzzy solving technology and SAAFSA is proposed.

In practical applications, there are three or more optimization objectives for many problems. For the target space with high-dimensional property, the existing multiobjective evolutionary algorithm has the following problems in the calculation [26]:

(1) The increase in the number of optimization goals leads to an increase in the proportion of nondominated solutions in the solution set, resulting in a slow search process

(2) For the space of high-dimensional objects, the index of maintaining population diversity has high computational complexity, and it is difficult to find the adjacent elements of the solution

(3) For the space of high-dimensional targets, the searchability of the reorganization operator is too inefficient

(4) Due to a large number of objective functions, the Pareto frontier is difficult to express intuitively, making it difficult for different decision makers to choose the solution they need

(5) The calculation cost of the performance index of the solution is too high, and the advantages and disadvantages of the calculation result are not easy to evaluate

(6) For the high-dimensional target space, how to visualize the results is also a difficult problem

For the first three problems, they can be alleviated by transforming the multiobjective evolutionary algorithm, but the last three problems have not yet been solved well. In addition to the above problems, in the actual problem, because the target solution set is concentrated on the local area of the frontier of Pareto, how to find the global optimal solution is also a difficulty of applying the algorithm to the actual problem.

In a great number of meta-heuristic algorithms, the nondominated sorting genetic algorithm (NSGA) is widely used in different problems and obtains a good application effect [27]. NSGA-III and NSGA-II have similar frames, and the differences between them mainly lie in the change of the selection mechanism, wherein NSGA-II is mainly sorted by relying on congestion, and its role in the high-dimensional target space is not obvious, but NSGA-III has rearranged the sorting of congestion and introduced widely distributed reference points to maintain the diversity of the population [28].

\subsection{Solution Algorithm Based on NSGA-III}

4.1.1. Coding and Decoding Approach. When using a genetic algorithm to solve the problem, different coding approaches have a direct influence on solving the efficiency of the problem. According to the characteristics of two-sided matching, two kinds of different coding approaches can be designed.
If $m=n$, the chromosome with the length of $m$ or $n$ can be designed and presented in the way of a one-dimensional array. If the values of $m$ and $n$ are both 8 , and the two sides to be matched are 1-to-1, the following encoding method can be given as shown in Figure 1.

If $m \neq n$, that is, the code lengths of the supply and demand sides are inconsistent, then the two different cases $m>n$ and $m<n$ can be designed into the same nonequal length encoding method. Assume $m=8$ and $n=6$; the long coding approach of designing the chromosome is shown in Figure 2.

The short coding approach of designing the chromosome is shown in Figure 3.

Essentially speaking, the effects of the three coding approaches are the same. Without loss of generality, in solving this problem, we assume $m \neq n$ and adopt a long coding approach.

The process of decoding chromosomes adopting a long coding approach is very simple. Taking Figure 2 as the example, when decoding the chromosome, if $A_{1}$ and $B_{3}$ match with each other, the value of $x_{13}$ is 1 , and if $A_{2}$ and $B_{2}$ match with each other, the value of $x_{22}$ is 1 . In a similar way, if $A_{3}$ and $B_{5}$ match with each other, $A_{4}$ and $B_{4}$ match with each other. Each objective value can be calculated according to the value of $x_{i j}$.

4.1.2. Crossover Operation. The goal of crossover operation is to increase the diversity of the chromosome so as to further extend the solution space. This paper designs two crossover approaches; one is to take crossover operation of equal position exchange to the two chromosomes, and the specific operation approach is shown in Figure 4.

Secondly, use the individual chromosome, in the first half or the second half of the chromosome, select the gene ordinal with the length less than the 1/2 chromosome, and realize the equal length exchange of the front and back of itself; the specific operation is shown in Figure 5.

4.1.3. Mutation Operation. The goal of mutation operation is to increase the diversity of the solution; here, design two approaches of mutation operation. One is to select two different genes on the chromosome in allusion to a selected paternal chromosome and then exchange the position of the two genes; the specific operation is shown in Figure 6.

Secondly, on the selected chromosome, select one or some gene loci randomly, and change their corresponding gene value; the specific operation is shown in Figure 7.

4.1.4. Chromosome Repair Mechanism. After crossing or mutating chromosomes, a nonfeasible chromosome can be produced. As shown in the chromosome in Figure 4, after the exchange and crossover of two chromosomes, $C_{1}$ contains three service objects 5 , but lacks service object 1 ; according to constraint equations (6)-(9), we can know that this chromosome is illegal and needs to be processed. In allusion to the processing of the nonfeasible chromosome, there are generally three operations as follows [29]: 


\begin{tabular}{|c|c|c|c|c|c|c|c|}
\hline $\begin{array}{ll}A_{i} & 1\end{array}$ & 2 & 3 & 4 & 5 & 6 & 7 & 8 \\
\hline 3 & 2 & 7 & 4 & 8 & 6 & 5 & 1 \\
\hline
\end{tabular}

(a)

\begin{tabular}{|c|c|c|c|c|c|c|c|c|}
\hline$B_{j}$ & 1 & 2 & 3 & 4 & 5 & 6 & 7 & 8 \\
\hline$A_{i}$ & 3 & 2 & 7 & 4 & 8 & 6 & 5 & 1 \\
\hline
\end{tabular}

(b)

Figure 1: Equal length coding approach of two-sided matching of supply and demand. (a) Coding approach 1. (b) Coding approach 2.

\begin{tabular}{c|c|c|c|c|c|c|c|c|}
$A_{i} A_{i}$ & 1 & 2 & \multicolumn{1}{c}{3} & \multicolumn{1}{c}{4} & \multicolumn{1}{l}{5} & 6 & 7 & 8 \\
\cline { 2 - 9 }$B_{j}$ & 3 & 2 & 5 & 4 & 3 & 6 & 5 & 1 \\
\hline
\end{tabular}

FIGURE 2: Long coding approach of two-sided matching of supply and demand.

\begin{tabular}{l|l|l|l|l|l|l|}
$A_{i}$ & 1 & 2 & \multicolumn{1}{l}{3} & 4 & 5 & 6 \\
\hline$B_{j}$ & 8 & 2 & 5 & 4 & 3 & 7 \\
\hline
\end{tabular}

FIGURE 3: Short coding approach of two-sided matching of supply and demand.

\begin{tabular}{|c|c|c|c|c|c|c|c|c|}
\hline$P_{1}$ & 1 & 2 & 3 & 4 & 5 & 6 & 7 & 8 \\
\hline$B_{j}$ & 3 & 2 & 4 & 6 & 5 & 6 & 5 & 1 \\
\hline \multirow{2}{*}{$P_{2}$} & 1 & 2 & 3 & 4 & 5 & 6 & 7 & 8 \\
\hline & 4 & 1 & 3 & 6 & 6 & 5 & 2 & 5 \\
\hline \multirow{2}{*}{$C_{1}$} & 1 & 2 & 3 & 4 & 5 & 6 & 7 & 8 \\
\hline & 3 & 2 & 4 & 6 & 5 & 5 & 2 & 5 \\
\hline \multirow{2}{*}{$C_{2}$} & 1 & 2 & 3 & 4 & 5 & 6 & 7 & 8 \\
\hline & 4 & 1 & 3 & 6 & 6 & 6 & 5 & 1 \\
\hline
\end{tabular}

Figure 4: Crossover operation of an equal position of the two chromosomes.

\begin{tabular}{|c|c|c|c|c|c|c|c|c|}
\hline$A_{i}$ & 1 & 2 & 3 & 4 & 5 & 6 & 7 & 8 \\
\hline${ }^{P_{1}} \quad B_{j}$ & 3 & 2 & 4 & 6 & 5 & 6 & 5 & 1 \\
\hline$A_{i}$ & 1 & 2 & 3 & 4 & 5 & 6 & 7 & 8 \\
\hline$C_{1}$ & 3 & 5 & 6 & 5 & 6 & 4 & 2 & 1 \\
\hline
\end{tabular}

Figure 5: Crossover operation of individual chromosome symmetry.

(1) Repair mechanism: for some nonfeasible chromosomes, different repair strategies are designed according to the constraints, so as to make them move to the nearest feasible space. For a different problem, the designed repair mechanism is different,

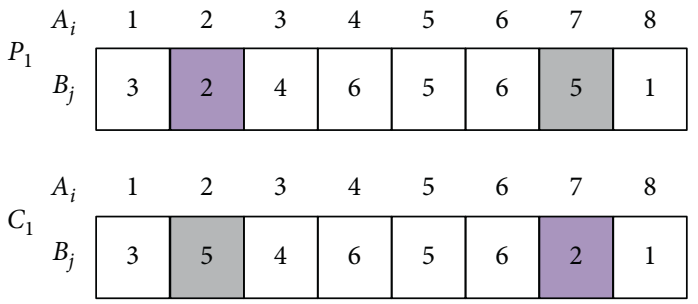

Figure 6: Mutation of two-point exchange.

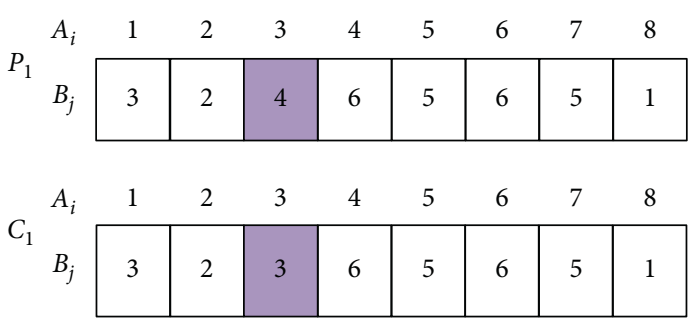

FIGURE 7: Single point mutation.

this approach is very time-consuming, and the repair process is complex.

(2) Punishment mechanism: set penalty conditions according to the objective function of the problem and degree of violation of constraints, which can reduce the probability of this chromosome to be selected but can increase the searching scope of the solution to some extent.

(3) Directly discarded: do not improve the nonfeasible solution and directly discard it, which is the simplest way but will lead to the loss of diversity of the solution.

In this paper, we design the repair strategy of the chromosome. From the features of the design of the chromosome, we know that the existence of an illegal chromosome is due to the loss of part of the supply individual, but the quantity of part of the supply individual is excessive. Therefore, design the repair strategy to be specific as the following:

Step 1: traverse the individual chromosome, and do some statistics for the code and quantity of the supply individual

Step 2: if the lost supply individuals exist, select one from the individuals with supply statistics quantity of more than 2 randomly, and displace the lost individuals 
Step 3: check the legality of chromosomes; if it is still illegal, repeat to return to Step 1; otherwise, the repair is successful

4.1.5. The Operation of Selection. In the NSGA-III algorithm, the $t$-th step is produced. $P_{t}$ is the $t$-th parent whose size is $N$, and its generated offspring is $Q_{t}$ whose size is $N$, too. Firstly, combine the parent and offspring with $R_{t}=P_{t} \cup Q_{t}$, the size of $R_{t}$ is $2 N$, and there is a need to select $N$ individuals from it. In order to achieve this selection process, through the nondominant sorting strategy, divide $R_{t}$ into multiple nondominant layers $F_{1}, F_{2}, F_{3}, \ldots$ and then combine with the number of individuals in each layer to begin to build a new population $S_{t}$ until its size is $N$ or exceeds $N$ for the first time; then, the last layer is called the $l$ th layer. The solutions of the $l+1$ th layer and the above layers will be eliminated. In most cases, the last layer is accepted by the only part of the acceptable layer ( $l$ th layer). In this case, diversity is used to measure the solution in $l$ layers so as to make selections [26].

In NSGA-II, the selection of this part of individuals uses congestion sorting of the individuals in the same layer; in NSGA-III, the selection of individuals is based on the approach of reference point. In NSGA-III, in allusion to the selection of solution in the $l$ th layer, the approach based on the reference point is used; in order to solve the multiobjective optimization problem with three or more objectives, if we continue to use congestion distance, the convergence and diversity of the algorithm are worse.

NSGA-III is applied to the matching of the supply side and the demand side of logistics service based on uncertain preference ordinal information, and the process of the algorithm is as shown in Figure 8.

\section{Analysis of Calculation Examples and Results}

An intermediary website of logistics service provides service in regard to the supply side and the demand side of logistics service; now, there are 5 demand sides of logistics service $\mathbf{A}=\left(A_{1}, A_{2}, A_{3}, A_{4}, A_{5}\right)$, which demand logistics service; after releasing information on the intermediary website, there are 6 companies of logistics service $\mathbf{B}=\left(B_{1}, B_{2}, B_{3}, B_{4}, B_{5}, B_{6}\right)$, which register to provide logistics service. The demand side $A_{i}$ of logistics service makes comprehensive evaluation to the supply side of logistics service from the price, responsiveness, on-time delivery rate, cargo damage rate, and so on and provides the uncertain preference ordinal vector $\widetilde{O c}=\left\{\widetilde{o c_{i 1}} \widetilde{o c_{i 2}}, \ldots, \widetilde{o c_{i n}}\right\}$ of subject set $\mathbf{A}$ of the demand side of logistics service, see Table 1 for the specific value.

The supply side of logistics service provides the uncertain preference ordinal vector $\widetilde{o d}=\left\{\widetilde{o d_{1 j}} \widetilde{o d_{2 j}}, \ldots, \widetilde{o d_{m j}}\right\}$ to the demand side of logistics service in the aspects of cargo quantity, payback period, return rate, enterprise size, and so on, see Table 2 for a specific value.

According to the preference ordinal vector provided in Tables 1 and 2, match them with the interval number; the

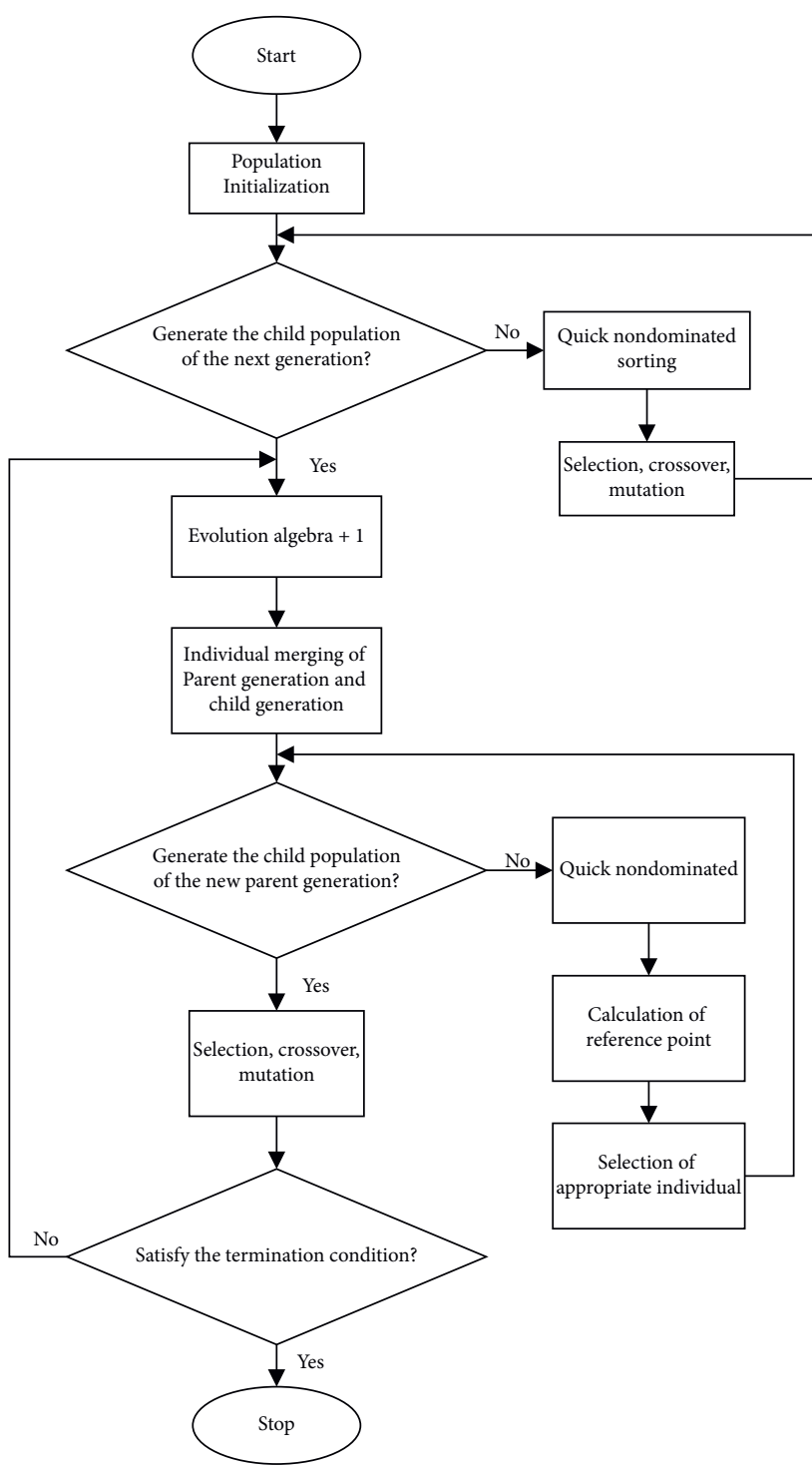

FIgURe 8: Algorithm flowchart.

TABLE 1: Uncertain preference ordinal vector $\widetilde{O c}$ of the demand side to the supply side.

\begin{tabular}{ccccccc}
$\widetilde{\widetilde{o c}_{i j}}$ & $B_{1}$ & $B_{2}$ & $B_{3}$ & $B_{4}$ & $B_{5}$ & $B_{6}$ \\
\hline$A_{1}$ & {$[3,4]$} & {$[1,1]$} & {$[5,6]$} & {$[4,5]$} & {$[1,2]$} & {$[3,3]$} \\
$A_{2}$ & {$[4,6]$} & {$[2,3]$} & {$[4,5]$} & {$[3,4]$} & {$[4,5]$} & {$[1,2]$} \\
$A_{3}$ & {$[3,3]$} & {$[5,5]$} & {$[2,2]$} & {$[6,6]$} & {$[1,1]$} & {$[4,4]$} \\
$A_{4}$ & {$[5,5]$} & {$[1,4]$} & {$[1,2]$} & {$[2,4]$} & {$[5,6]$} & {$[3,4]$} \\
$A_{5}$ & {$[1,1]$} & {$[3,3]$} & {$[5,5]$} & {$[2,2]$} & {$[4,4]$} & {$[6,6]$} \\
\hline
\end{tabular}

TABLE 2: Uncertain preference ordinal vector $\widetilde{D}_{i}$ of the supply side to the demand side.

\begin{tabular}{lccccc}
\hline${\widetilde{o d_{i j}}}$ & $A_{1}$ & $A_{2}$ & $A_{3}$ & $A_{4}$ & $A_{5}$ \\
\hline$B_{1}$ & {$[4,5]$} & {$[3,5]$} & {$[2,3]$} & {$[1,2]$} & {$[5,5]$} \\
$B_{2}$ & {$[1,1]$} & {$[3,3]$} & {$[5,5]$} & {$[2,2]$} & {$[4,4]$} \\
$B_{3}$ & {$[1,3]$} & {$[5,5]$} & {$[2,3]$} & {$[4,5]$} & {$[3,5]$} \\
$B_{4}$ & {$[3,5]$} & {$[2,3]$} & {$[4,5]$} & {$[5,5]$} & {$[1,2]$} \\
$B_{5}$ & {$[2,4]$} & {$[1,3]$} & {$[5,5]$} & {$[3,5]$} & {$[4,5]$} \\
$B_{6}$ & {$[1,2]$} & {$[2,4]$} & {$[1,1]$} & {$[2,3]$} & {$[4,5]$} \\
\hline
\end{tabular}


greater value indicates that the sort is later in the selection. In order to calculate the uncertain satisfaction of the supply and demand sides, the preference ordinal vector needs to be converted into satisfaction. According to the characteristics of the case, in this paper, assume $M C=7$, let $\widetilde{c_{i j}}=M C-\widetilde{o c_{i j}}$, and obtain uncertain satisfaction matrix $\widetilde{C}$ of the demand-side subject set $A$ to the supply-side subject set $B$, as shown in Table 3 .

Similarly, assume $M D=6$, let $\widetilde{d_{i j}}=M D-\widetilde{o d}_{i j}$, and obtain uncertain satisfaction matrix $\widetilde{D}$ of the supply-side subject set $B$ to the demand-side subject set $A$, as shown in Table 4.

According to the satisfaction in Tables 3 and 4, the size of the interval number is compared by using formula (1) to determine the dominance relationship between the solutions.

5.1. Calculation Results and Analysis. Firstly, set the size of the population as popsize $=200$; the maximum number of iterations is 300 , the crossover probability is 0.8 , the selection probability of the two crossover modes is 0.5 , the mutation probability is 0.3 , and the selection probability of the two mutation modes is 0.5 .

Secondly, construct the initial population, and the construction step is as follows:

Step 1: make the corresponding code for each individual in $\mathbf{B}=\left(B_{1}, B_{2}, B_{3}, B_{4}, B_{5}, B_{6}\right)$, wherein the corresponding code for $B_{6}$ is the random number between $[1,5]$

Step 2: in regard to $\left(B_{1}, B_{2}, B_{3}, B_{4}, B_{5}, B_{6}\right)$, two different numbers $i_{1}$ and $i_{2}$ are randomly generated between [1, $6]$, and the corresponding code of $B_{i_{1}}$ and $B_{i_{2}}$ is exchanged; the step is repeated for $M$ times

Step 3: repeat Steps 1 and 2 until the quantity of population is reached

After the algorithm is done, the nondominated solution set is obtained. Take the matching relation of the first 5 approximate optimal solutions of each optimization objective and their corresponding objective value so as to make comparison and analysis.

In the generated Pareto solution set, make sorting of objective 1 according to equation (1), and the first 5 approximate optimal solutions and their corresponding objective value are shown in Table 5 .

From the data in Table 5, we can know that when the demand side selects the supply side according to its uncertain preference ordinal, the differences of each matching result is not large, take the solution 1 and 2 for example, the demand 1 selects the supply 2, the demand 2 selects the supply 6 , the demand 3 selects the supply 5 , the demand 4 selects the supply 3 , the demand 5 selects the supply 1 ; the difference is that in solution 1, the supply 4 selects the demand 5 , and in solution 2 , the supply 4 selects the demand 4. From solution 4 and 5 , we can know that, though the infimum of the corresponding interval number of the objective 1 of the solution 5 is smaller, the infimum of the
TABLE 3: Uncertain satisfaction $\widetilde{C}$ of the demand side to the supply side.

\begin{tabular}{ccccccc}
\hline$\widetilde{c_{i j}}$ & $B_{1}$ & $B_{2}$ & $B_{3}$ & $B_{4}$ & $B_{5}$ & $B_{6}$ \\
\hline$A_{1}$ & {$[3,4]$} & {$[6,6]$} & {$[1,2]$} & {$[2,3]$} & {$[5,6]$} & {$[4,4]$} \\
$A_{2}$ & {$[1,2]$} & {$[4,5]$} & {$[2,3]$} & {$[3,4]$} & {$[2,3]$} & {$[5,6]$} \\
$A_{3}$ & {$[4,4]$} & {$[2,2]$} & {$[5,5]$} & {$[1,1]$} & {$[6,6]$} & {$[3,3]$} \\
$A_{4}$ & {$[2,2]$} & {$[3,6]$} & {$[5,6]$} & {$[3,5]$} & {$[1,2]$} & {$[3,4]$} \\
$A_{5}$ & {$[6,6]$} & {$[4,4]$} & {$[2,2]$} & {$[5,5]$} & {$[3,3]$} & {$[1,1]$} \\
\hline
\end{tabular}

TABLE 4: Uncertain satisfaction $\widetilde{D}$ of the supply side to the demand side.

\begin{tabular}{cccccc}
\hline$\widetilde{d_{i j}}$ & $A_{1}$ & $A_{2}$ & $A_{3}$ & $A_{4}$ & $A_{5}$ \\
\hline$B_{1}$ & {$[1,2]$} & {$[1,3]$} & {$[3,4]$} & {$[4,5]$} & {$[1,1]$} \\
$B_{2}$ & {$[5,5]$} & {$[3,3]$} & {$[1,1]$} & {$[4,4]$} & {$[2,2]$} \\
$B_{3}$ & {$[3,5]$} & {$[1,1]$} & {$[3,4]$} & {$[1,2]$} & {$[1,3]$} \\
$B_{4}$ & {$[1,3]$} & {$[3,4]$} & {$[1,2]$} & {$[1,1]$} & {$[4,5]$} \\
$B_{5}$ & {$[2,4]$} & {$[3,5]$} & {$[1,1]$} & {$[1,3]$} & {$[1,2]$} \\
$B_{6}$ & {$[4,5]$} & {$[2,4]$} & {$[5,5]$} & {$[3,4]$} & {$[1,2]$} \\
\hline
\end{tabular}

TABLE 5: Approximate optimal solutions of objective 1.

\begin{tabular}{|c|c|c|c|c|c|}
\hline No. & Solution & $\begin{array}{c}\text { Objective } \\
1\end{array}$ & Objective 2 & $\begin{array}{c}\text { Objective } \\
3\end{array}$ & Objective 4 \\
\hline $1-1$ & $\begin{array}{c}5,1,4,5, \\
3,2\end{array}$ & {$[33,35]$} & {$[1.16,2.04]$} & {$[14,18]$} & $\begin{array}{l}{[15.33,} \\
18.00]\end{array}$ \\
\hline $1-2$ & $\begin{array}{c}5,1,4,4 \\
3,2\end{array}$ & {$[31,35]$} & {$[1.16,7.64]$} & {$[11,14]$} & $\begin{array}{l}{[12.83,} \\
15.33]\end{array}$ \\
\hline $1-3$ & $\begin{array}{c}5,1,4,2 \\
3,2\end{array}$ & {$[31,34]$} & {$[3.76,7.64]$} & {$[13,17]$} & $\begin{array}{l}{[12.83,} \\
14.83]\end{array}$ \\
\hline $1-4$ & $\begin{array}{c}3,1,4,5 \\
1,2\end{array}$ & {$[30,33]$} & {$[2.96,4.04]$} & {$[17,24]$} & $\begin{array}{c}{[10.83,} \\
6.00]\end{array}$ \\
\hline $1-5$ & $\begin{array}{c}5,4,3,5 \\
1,2\end{array}$ & {$[29,34]$} & {$[1.76,5.96]$} & {$[16,22]$} & {$[7.33,9.33]$} \\
\hline
\end{tabular}

corresponding interval number of the objective 1 of the solution 4 is greater, but the sorting result is that $[30,33]$ is ranked ahead of $[29,34]$, because the width of the corresponding objective 1 of the solution 5 is greater, then its uncertainty is greater, according to the sorting rule, its sorting ordinal ranks latter.

In the obtained Pareto solution set, make sorting of the objective 2 according to equation (1), and the first 5 approximate optimal solutions and their corresponding objective value are shown in Table 6 .

From the data in Table 6, we can know that the sorting of the interval number with greater uncertainty is latter; in the approximate optimal solutions corresponding to objective 2 , part of the solution is also the approximate optimal solutions corresponding to objective 1 . From the point of view of the demand side, this matching ordinal is better and has a bigger change to be selected.

Similarly, in the obtained Pareto solution set, make sorting of objective 3 according to equation (1), and the first 5 approximate optimal solutions and their corresponding objective value are shown in Table 7 . 
TABLE 6: Approximate optimal solutions of objective 2.

\begin{tabular}{|c|c|c|c|c|c|}
\hline No. & Solution & Objective 1 & Objective 2 & Objective 3 & Objective 4 \\
\hline $2-1$ & $5,1,4,5,3,2$ & {$[33,35]$} & {$[1.16,2.04]$} & {$[14,18]$} & {$[15.33,18.00]$} \\
\hline $2-2$ & $3,2,4,5,1,2$ & {$[28,32]$} & {$[2.64,4.00]$} & {$[15,22]$} & {$[5.33,5.50]$} \\
\hline $2-3$ & $3,1,4,5,1,2$ & {$[30,33]$} & {$[2.96,4.04]$} & {$[17,24]$} & {$[6.00,10.83]$} \\
\hline $2-4$ & $5,4,3,5,1,2$ & {$[29,34]$} & {$[1.76,5.96]$} & {$[16,22]$} & {$[7.33,9.33]$} \\
\hline $2-5$ & $5,1,4,4,3,2$ & {$[31,35]$} & {$[1.16,7.64]$} & {$[11,14]$} & {$[12.83,15.33]$} \\
\hline
\end{tabular}

TABLE 7: Approximate optimal solutions of objective 3.

\begin{tabular}{|c|c|c|c|c|c|}
\hline No. & Solution & Objective 1 & Objective 2 & Objective 3 & Objective 4 \\
\hline $3-1$ & $4,1,3,5,2,1$ & {$[24,25]$} & {$[12.76,16.16]$} & {$[23,29]$} & {$[0.83,2.83]$} \\
\hline $3-2$ & $4,4,3,5,2,1$ & {$[21,25]$} & {$[12.44,12.76]$} & {$[22,28]$} & {$[1.33,1.33]$} \\
\hline $3-3$ & $3,1,3,5,2,4$ & {$[25,27]$} & {$[7.00,12.76]$} & {$[21,27]$} & {$[1.50,3.50]$} \\
\hline $3-4$ & $4,1,3,5,1,2$ & {$[28,30]$} & {$[10.64,12.96]$} & {$[20,27]$} & {$[1.50,7.33]$} \\
\hline $3-5$ & $3,4,1,5,2,4$ & {$[18,24]$} & {$[12.16,13.84]$} & {$[20,27]$} & {$[1.33,1.50]$} \\
\hline
\end{tabular}

From the data in Table 7, we can know that compared with the result of Table 5, the matching result is greatly changed, presenting that the decision-making goal of the demand side and the supply side is not consistent; for the corresponding optimal matching result of objective 3 , the sorting of its corresponding objective 1 is worse, and the variance of corresponding objective 2 is greater.

In the obtained Pareto solution set, make sorting of objective 4 according to equation (1), and the first 5 approximate optimal solutions and their corresponding objective value are shown in Table 6 .

From the data in Table 8, we can know that when the variance of the selection result pursued by the supply side is smaller, the difference of its approximate optimal solutions and the corresponding approximate optimal solutions of objective 3 is not greater, and the supply side can make comprehensive decision-making by combining with the data in Tables 7 and 8; the infimum and supremum of the interval number corresponding to objective 4 in solution $4-1$ are the same, and this value is a determined number; the infimum of objective 4 in solution $4-3$ is 0 , but the value of its supremum is greater, indicating that the uncertainty of this interval number is greater.

5.2. Sensitivity Analysis of Algorithm Parameters. In the designed algorithm, the choice of different parameters has a great influence on the performance of the algorithm. When evaluating the performance of an algorithm, the following two aspects are usually considered. One is the closeness of the set of nondominated solutions to the Pareto-optimal front; the other is the diversity of the Pareto front distribution [30].

In this paper, the interval number is used to study the matching problem between the supplier and the demander. It is difficult to calculate the closeness between the set of nondominated solutions and the Pareto-optimal front. Therefore, this paper chooses the diversity of the Pareto front distribution as an indicator, uses the
TABle 8: Approximate optimal solutions of objective 4.

\begin{tabular}{cccccc}
\hline No. & Solution & Objective 1 & Objective 2 & Objective 3 & Objective 4 \\
\hline $4-1$ & $4,4,3,5,2,1$ & {$[21,25]$} & {$[12.44,12.76]$} & {$[22,28]$} & {$[\mathbf{1 . 3 3}, \mathbf{1 . 3 3}]$} \\
$4-2$ & $3,4,1,5,2,4$ & {$[18,24]$} & {$[12.16,13.84]$} & {$[20,27]$} & {$[\mathbf{1 . 3 3} \mathbf{1 . 5 0}]$} \\
$4-3$ & $2,3,5,1,4,5$ & {$[9,13]$} & {$[8.44,8.76]$} & {$[6,15]$} & {$[\mathbf{0 . 0 0}, \mathbf{3 . 5 0}]$} \\
$4-4$ & $4,1,3,5,2,1$ & {$[24,25]$} & {$[12.76,16.16]$} & {$[23,29]$} & {$[\mathbf{0 . 8 3}, \mathbf{2 . 8 3}$} \\
$4-5$ & $1,2,4,3,5,5$ & {$[17,20]$} & {$[17.00,24.56]$} & {$[8,13]$} & {$[\mathbf{0 . 8 3}, \mathbf{3 . 3 3}]$} \\
\hline
\end{tabular}

number of the set of nondominated solutions to analyze the performance of the algorithm under different parameters, and combines the calculation time to analyze the calculation efficiency of the algorithm under different parameters.

When analyzing the sensitivity of the parameters in the algorithm, under the premise of a fixed number of iterations $(I=200)$, by setting different values of population size, cross probability, and variation probability, we get the number of nondominated solutions and the corresponding calculation time. Calculate 10 times under each set of parameters, and the average data obtained are shown in Table 9.

From the data in Table 9, we can know that the number of nondominated solutions and the calculation time of the algorithm are mainly related to the population size; when the population size is the same, the cross probability and the variation probability have an impact on the number of nondominated solutions and the calculation time of the algorithm, but the difference is not significant; when the population size is 400 and 1000, respectively, there is a significant difference in the calculation time of the algorithm, but the number of nondominated solutions generated does not change much, which is related to the selected data set; because the instance data in this article are relatively small, the number of nondominated solutions obtained is limited.

It can be seen from the above analysis that different population sizes can be set for different scale calculation requirements to achieve a balance between solution results and solution efficiency. 
TABLE 9: Algorithm performance under different parameters.

\begin{tabular}{lcccc}
\hline Population size & Cross probability & Variation probability & Average number of nondominated solutions & Average calculation time (s) \\
\hline 100 & 0.8 & 0.2 & 12.2 & 1.614 \\
& 0.8 & 0.6 & 14.7 & 1.623 \\
& 0.2 & 0.2 & 11.3 & 1.606 \\
400 & 0.2 & 0.6 & 12.3 & 1.616 \\
& 0.8 & 0.2 & 20.4 & 10.709 \\
& 0.8 & 0.6 & 22.6 & 10.808 \\
1000 & 0.2 & 0.2 & 20.8 & 10.456 \\
& 0.2 & 0.6 & 21.3 & 33.125 \\
& 0.8 & 0.2 & 22.9 & 33.687 \\
& 0.8 & 0.6 & 23.2 & 32.587 \\
& 0.2 & 0.2 & 21.2 & 33.144 \\
\hline
\end{tabular}

\section{Conclusion and Future Research}

This paper provides the research thought of this problem based on the decision-making problem of two-sided matching of supply and demand of logistics service based on the uncertain preference ordinal and provides the general research solution of solving the problem based on the decision-making problem of two-sided matching of supply and demand of logistics service based on the uncertain preference ordinal. In this paper, the uncertain preference ordinal vectors of the supply and demand sides are expressed by interval numbers, which can meet the preference ordinal setting requirements of the supply and demand sides in practical applications and can also be characterized by appropriate mathematical expressions. On this basis, the uncertain preference ordinal is calculated according to the calculation rules of the interval number, and the interval number vector can be transformed to obtain the sorted satisfaction. Considering the overall satisfaction and individual satisfaction of both the demand side and the supply side of logistics services, a multiobjective planning model is built, and the solution is solved on the basis of NSGA-III; the coding approach, genetic operation, and repair strategy are designed according to the features of the problem, and the matching result of supply and demand of logistics service based on the uncertain preference ordinal can be obtained after the solution. The following can be seen from the case results in the paper: (1) considering the balance of overall satisfaction and individual satisfaction at the same time, it can better characterize the interest relationship between the supplier and the demander in the logistics service and guide the decision maker to obtain a satisfactory and stable match between the supplier and the demander. (2) Satisfy decision makers with different preferences for overall and individual satisfaction and guide decision makers to obtain a satisfactory matching relationship between the supplier and the demander. This article considers the uncertainty of the actual impact of the individual supply and demand sides, the uncertainty problem is clearly treated using the interval number theory and the interval number comparison method, and effective calculation results are obtained to solve the two-sided matching problem of logistics service supply and demand so that the solved problem of the supply and demand of logistics services is more suitable for the actual needs. Through the sensitivity analysis of algorithm parameters, it can be known that, for different scales of calculation requirements, different population sizes can be set to achieve a balance between solution results and solution efficiency.

There are still many issues which need to be resolved in the future: (1) the two-sided matching decision-making problem of logistics service supply and demand based on the preference ordinal and the mixed preference ordinal in an uncertain environment is a new research topic with a broad field of exploration, and further research in theory, method, and application is needed. (2) The optimization decision model can also be used to conduct in-depth research and analysis on theoretical issues such as robustness and the existence of optimal solutions in the two-sided matching algorithm.

\section{Data Availability}

All the data used to support the findings of this study are included within the article.

\section{Conflicts of Interest}

The authors declare that there are no conflicts of interest regarding the publication of this paper.

\section{References}

[1] D. Gale and L. S. Shapley, "College admissions and the stability of marriage," The American Mathematical Monthly, vol. 69, no. 1, pp. 9-15, 1962.

[2] Y. Qi and Y. Fan, "Research on decision-making problem of two-sided matching of supply and demand of logistics service based on uncertain preference ordinal information," Operations Research and Management Science, vol. 21, no. 1, pp. 57-63, 2012.

[3] Y. Qi, L. Zhang, and L. Zhang, "Decision-making approach of two-sided matching considering the psychological behavior of the body under uncertain preference ordinal information," Operations Research and Management Science, vol. 24, no. 2, pp. 113-120, 2015.

[4] V. Kayvanfar, S. M. M. Husseini, B. Karimi, and M. S. Sajadieh, "Bi-objective intelligent water drops algorithm to a practical multi-echelon supply chain optimization problem," Journal of Manufacturing Systems, vol. 44, no. 1, pp. 93-114, 2017. 
[5] T. Sun, D. Zhang, G. Huang, C. Ye, and Q. Liangqi, "Twosided matching approach with multiple forms of preference information," Fuzzy Systems and Mathematics, vol. 31, no. 4, pp. 108-116, 2017.

[6] V. Kayvanfar, S. M. Moattar Husseini, M. S. Sajadieh, and B. Karimi, "A multi-echelon multi-product stochastic model to supply chain of small-and-medium enterprises in industrial clusters," Computers \& Industrial Engineering, vol. 115, pp. 69-79, 2018.

[7] Z.-P. Fan, M.-Y. Li, and X. Zhang, "Satisfied two-sided matching: a method considering elation and disappointment of agents," Soft Computing, vol. 22, no. 21, pp. 7227-7241, 2018.

[8] V. Kayvanfar, M. S. Sajadieh, S. M. Moattar Husseini, and B. Karimi, "Analysis of a multi-echelon supply chain problem using revised multi-choice goal programming approach," Kybernetes, vol. 47, no. 1, pp. 118-141, 2018.

[9] Y. Qi, "Decision-making approach of two-sided matching based on incomplete score information," Systems Engineering, vol. 31, no. 9, pp. 79-83, 2013.

[10] D. Zhang, T. Sun, G. Huang, C. Ye, and L. Wan, "Decisionmaking model of two-sided matching based on Borda approach uncertain preference ordinal," Statistics \& Information Forum, vol. 32, no. 12, pp. 22-26, 2017.

[11] T. You, Z. Fan, and Z. Yu, "An assignment method for group decision making with uncertain preference ordinals," Journal of Systems Science and Systems Engineering, vol. 21, no. 2, pp. 174-183, 2012.

[12] X. Liu and H. Ma, "A two-sided matching decision model based on uncertain preference sequences," Mathematical Problems in Engineering, vol. 2015, Article ID 241379, 10 pages, 2015.

[13] V. Kayvanfar, S. M. Moattar Husseini, B. Karimi, and M. S. Sajadieh, "Supply-demand hub in industrial clusters: a stochastic approach," Engineering Optimization, vol. 50, no. 9, pp. 1561-1577, 2018.

[14] E. B. Tirkolaee, A. Mardani, Z. Dashtian, M. Soltani, and G.-W. Weber, "A novel hybrid method using fuzzy decision making and multi-objective programming for sustainablereliable supplier selection in two-echelon supply chain design," Journal of Cleaner Production, vol. 250, 2020.

[15] V. Kayvanfar, S. M. Moattar Husseini, Z. NengSheng, B. Karimi, and M. S. Sajadieh, "A practical supply-demand hub in industrial clusters: a new perspective," Management Research Review, vol. 42, no. 1, pp. 68-101, 2019.

[16] R. E. Moorse, Interval Analysis, PrenticeHall, Englewood Cliffs, NJ, USA, 1966.

[17] R. E. Moorse, Methods and Applications of Interval Analysis, SIAM, Philadelphia, PA, USA, 1979.

[18] X. Zhang and X. Zhang, "The sorting of interval number and its application in the system decision-making," System Engineering Theory and Practice, vol. 19, no. 7, pp. 112-115, 1999.

[19] L. Liu, H. Mu, J. Yang, X. Li, and F. Wu, "A simulated annealing for multi-criteria optimization problem: DBMOSA," Swarm and Evolutionary Computation, vol. 14, no. 2, pp. 48-65, 2014.

[20] F. Szidarovsky, M. E. Gershon, and L. Dukstein, Techniques for Multiobjective Decision Making in Systems Management, Elsevier, New York, NY, USA, 1986.

[21] Y. Liu, Research on Decision-Making Approach of Two-Sided Matching with Individuals and Combination Preference Ordinal Information, Northeastern University, Boston, MA, USA, 2013.
[22] D. Kong and Y. Jiang, "Decision-making approach of twosided matching of personnel and post considering collaborative information," Operations Research and Management Science, vol. 27, no. 10, pp. 31-37, 2018.

[23] D. Yuan and Y. Jiang, "Stable two-sided matching model of patients and surgeons for elective surgery," System Engineering Theory and Practice, vol. 39, no. 7, pp. 1752-1762, 2019.

[24] A. K. Sangaiah, A. Goli, E. B. Tirkolaee, M. Ranjbar-Bourani, H. M. Pandey, and W. Zhang, "Big data-driven cognitive computing system for optimization of social media analytics," IEEE Access, vol. 8, pp. 82215-82226, 2020.

[25] E. B. Tirkolaee, A. Goli, and G.-W. Weber, "Fuzzy mathematical programming and self-adaptive artificial fish swarm algorithm for just-in-time energy-aware flow shop scheduling problem with outsourcing option," IEEE Transactions on Fuzzy Systems, p. 1, 2020.

[26] K. Deb and H. Jain, "An evolutionary many-objective optimization algorithm using reference-point-based nondominated sorting approach, part I: solving problems with box constraints," IEEE Transactions on Evolutionary Computation, vol. 18, no. 4, pp. 577-601, 2014.

[27] N. Srinivas and K. Deb, "Muiltiobjective optimization using nondominated sorting in genetic algorithms," Evolutionary Computation, vol. 2, no. 3, pp. 221-248, 1994.

[28] K. Deb, A. Pratap, S. Agarwal, and T. Meyarivan, "A fast and elitist multiobjective genetic algorithm: NSGA-II," IEEE Transactions on Evolutionary Computation, vol. 6, no. 2, pp. 182-197, 2002.

[29] C. A. C. Coello, "Constraint-handling techniques used with evolutionary algorithms," in Proceedings of the Companion on Genetic \& Evolutionary Computation Conference: Late Breaking Papers, Amsterdam, Netherlands, 2012.

[30] E. Zitzler, L. Thiele, M. Laumanns, C. M. Fonseca, and V. G. Da Fonseca, "Performance assessment of multiobjective optimizers: an analysis and review," IEEE Transactions on Evolutionary Computation, vol. 7, no. 2, pp. 117-132, 2003. 